\title{
Índice de colapsabilidad de la vena cava inferior como predictor de hipotensión intraoperatoria
}

\section{Inferior vena cava collapsibility index of the as a predictor of intraoperative hypotension}

María Celina Rossi MD1, Eduardo Pérez MD1,2, Silvana Montenegro Phd², Guillermina Harvey $\mathrm{Mg}^{2}$, Enzo Graziola Phd², Iván Groisman MD²,3

\begin{abstract}
Objective: To evaluate the lower vena cava Collapse Index $(\mathrm{CI})$ as a predictor parameter of hypotensive episodes after general anesthesia induction in ASA I and II patients who were scheduled for elective surgery. Materials and Methods: A prospective, observational and simple blind study was designed. A sample of 80 patients was recruited. In the preoperative stage, they underwent protocolarized sedation and $\mathrm{Cl}$ was obtained. Prior to induction, the baseline values of heart rate, non-invasive mean arterial pressure and continuous electrocardiographic tracing in DII were noted. In the post-orotracheal intubation stage, the aforementioned hemodynamic monitoring variables were recorded manually for 10 minutes. Results: The data of 78 individuals are presented. After anesthetic induction, 8 (10.3\%) patients developed hypotension. The adjustment of the univariate logistic regression model for $\mathrm{Cl}$ shows a good diagnostic capacity, with the area under the ROC curve equal to 0.76 . The chance of presenting hypotension is increased by $62 \%$ by increasing the $\mathrm{Cl}$ by 5 points $(p=0.003)$. Regarding the negative predictive value, we found that with values corresponding to the cutoff points between $39 \%$ and $46 \%$, a probability of at least $93.1 \%$ of not presenting hypotension was obtained. The optimal cutoff point of the $\mathrm{Cl}$ to predict hypotension is estimated at $43 \%$, with a sensitivity of $62.5 \%$ and a specificity of $92.9 \%$. Conclusion: A lower IC was associated with a lower probability of developing intraoperative hypotension. The use of this tool could be useful to anticipate which patients will be prone to intra-surgical hypotension.
\end{abstract}

\section{Key words:}

Inferior vena cava, hypotension, fluid therapy, perioperative care

Servicio de Anestesia, Analgesia y Reanimación, Hospital Provincial del Centenario, Santa Fe, Argentina.

Docente de la Carrera Posgrado en Anestesiología, Facultad de Ciencias Médicas, Universidad Nacional de Rosario, Argentina.

3 Hospital Privado de la Comunidad, Mar del Plata, Argentina.

Fecha de recepción: 04 de marzo de 2019

Fecha de aceptación: 12 de mayo de 2019

\section{ORCID}

https://orcid.org/0000-0003-2608-8277

Correspondencia:

María Celina Rossi

Email: celi_rossi@hotmail.com 


\section{RESUMEN}

Objetivo: Evaluar el índice de colapsabilidad (IC) de la vena cava inferior (VCI) como predictor de episodios hipotensivos posinducción de anestesia general en cirugía electiva de pacientes ASA I y II. Materiales y Métodos: Se diseñó un estudio prospectivo, observacional y simple ciego. Se reclutó una muestra de 80 pacientes. En etapa preoperatoria fueron sometidos a una sedación protocolizada y se obtuvo el IC. Previo a la inducción, se anotaron los valores basales de la frecuencia cardíaca, la presión arterial media y el trazado electrocardiográfico continuo en DII. Posintubación orotraqueal, se registraron manualmente las variables hemodinámicas mencionadas durante 10 minutos. Resultados: Se presentan datos de 78 individuos. Luego de la inducción, 8 $(10,3 \%)$ pacientes desarrollaron hipotensión. El ajuste del modelo de regresión logística univariado para IC muestra una buena capacidad diagnóstica, siendo el área bajo la curva ROC igual a 0,76. La chance de presentar hipotensión se incrementa en un $62 \%$ al aumentar el IC en 5 puntos $(p=0,003)$. En cuanto al valor predictivo negativo, encontramos que con valores correspondientes a los puntos de corte entre $39 \%$ y $46 \%$, se obtenía una probabilidad de $93,1 \%$ de no presentar hipotensión. El punto de corte óptimo del IC para predecir hipotensión se estima en $43 \%$, con una sensibilidad del $62,5 \%$ y una especificidad del 92,9\%. Conclusión: Un menor IC se asoció con una menor probabilidad de desarrollar hipotensión intraoperatoria. El uso de esta herramienta podría ser de utilidad para anticipar qué pacientes serán propensos a hipotensión intraquirúrgica.

\section{Palabras clave:}

Vena cava inferior, hipotensión,

fluidoterapia, cuidado perioperatorio

\section{Introducción}

$\mathrm{N}$ umerosos investigadores han intentado validar el uso de la ultrasonografía de la vena cava inferior $(\mathrm{VCl})$, pero las conclusiones no son unánimes en cuanto a su utilidad como herramienta para evaluar la volemia preoperatoria y como guía en la terapéutica de fluidos. Los parámetros estáticos clásicos, tales como la presión venosa central y la presión de oclusión de la arteria pulmonar, han sido extensamente criticados por su invasividad y falta de especificidad, mientras que los denominados parámetros dinámicos han sido propuestos como fácilmente obtenibles y no invasivos[1]-[14]. Tal como lo señalan Zhang y cols.[14], en su artículo respecto a esta temática, el rol de la evaluación preoperatoria del volumen intravascular en el desarrollo de hipotensión luego de la inducción, no ha sido suficientemente estudiado y frecuentemente esta última es comúnmente catalogada como clínicamente irrelevante a pesar de ser muy común en los pacientes sometidos a una anestesia general. En particular, luego de la inducción anestésica, los pacientes poseen un riesgo elevado de desarrollar hipotensión, debido a los efectos hemodinámicos depresores de los agentes anestésicos y a la falta de estímulo quirúrgico. Dado que la tensión arterial intraquirúgica puede ser controlada, debería constituir un target terapéutico importante, a diferencia de otras características y comorbilidades de los pacientes, raramente modificables[12]. El nivel de presión sanguínea que constituye un episodio severo y con facultad de provocar una insuficiencia renal aguda (IRA) o un infarto agudo de miocardio (IAM), permanece poco claro. Lo que sí se ha observado es que la lesión por isquemia reperfusión debida a hipotensión, puede sustancialmente contribuir a la IRA y al IAM[13].

La vena cava inferior es una vena de gran tamaño y fácilmente compresible. Su diámetro tiene una alta correlación con la función de las cavidades cardíacas derechas y su calibre no es afectado por la respuesta compensatoria vasoconstrictora que se genera ante la pérdida de volumen intravascular. Por este motivo, reflejaría el estatus de la volemia con mayor precisión que otros parámetros, como los basados en el sistema arterial (presión sanguínea, diámetro de la aorta, etc.)[7]. Por lo tanto, realizar el examen ultrasonográfico de la VCl previo a la anestesia general, con el objetivo de identificar a aquellos pacientes en riesgo de desarrollar hipotensión, constituye una excelente 
herramienta diagnóstica preoperatoria para planificar estrategias individualizadas en cuanto a la terapéutica con fluido e inotrópicos, para así evitar tanto el aporte innecesario de ambos como los episodios hipotensivos intraoperatorios[14],[15].

\section{Objetivo}

Evaluar la fiabilidad del índice de colapsabilidad de la VCl como parámetro dinámico y predictor de episodios hipotensivos posinducción de anestesia general en pacientes ASA I y II programados para cirugía electiva.

\section{Materiales y Métodos}

\section{Pacientes}

El presente estudio se llevó a cabo cumpliendo el código ético de la Organización Mundial de la Salud (Declaración de Helsinki) y fue aprobado por el Comité de Ética del Hospital Provincial del Centenario de la ciudad de Rosario para su ejecución. Se diseñó un estudio prospectivo, observacional y simple ciego, y se reclutó una muestra de pacientes programados para cirugía electiva de colecistectomía videolaparoscópica bajo anestesia general en el período comprendido entre octubre de 2017 hasta abril de 2018. Se incluyeron los pacientes que cumplieran los siguientes criterios: 1) adultos de entre 18 y 65 años; 2) ASA I o II; 3) ventilación espontánea e 4) índice de masa corporal (IMC) menor a 35. Por otro lado, los criterios de exclusión fueron: 1) arritmia cardiaca; 2) ascitis; 3) hipertensión abdominal; 4) cardiopatía valvular grave preexistente y/o derivación intracardíaca; 5) enfermedad vascular periférica; 6) angina cardiaca; 7) contraindicación para agentes sedantes y/o agentes bloqueantes neuromusculares; 8) desorden autonómico; 9) incompetencia mental; 10) vía aérea dificultosa; 11) tratamiento con inhibidores de la enzima convertidora de la angiotensina o bloqueantes de los receptores de angiotensina; 12) ayuno prolongado (mayor a $8 \mathrm{~h}$ ) y 13) semiología respiratoria patológica. Con respecto a este último punto y en orden de poder replicar los resultados de este estudio, la evaluación clínica de la semiología respiratoria de cada paciente (considerando que la magnitud de la excursión diafragmática y la fuerza inspiratoria pueden sesgar la fidelidad de los parámetros hemodinámicos relativos a la $\mathrm{VCI}$ ) se realizó en forma minuciosa. De este modo, todo paciente que posterior a la sedación en la sala de preanestesia presentó bradipnea, hipopnea, taquipnea o hiperpnea fue excluido del presente estudio. Se registraron además, los datos demográficos de dichos pacientes, incluyendo edad, sexo, altura y peso.

Se convocó a participar del estudio a todos los pacientes elegibles y que fueron legalmente aptos para dar su consentimiento informado.

\section{Variables hemodinámicas}

Todos los pacientes en etapa preoperatoria fueron sometidos a una sedación standard en la sala de preanestesia. Previo ingreso a la sala de operaciones, se obtuvo el Índice de colapsabilidad de la VCI con los pacientes posicionados en decúbito dorsal y a $0^{\circ}$.

Una vez en quirófano y precediendo la inducción anestésica, se anotaron los valores basales de los parámetros de monitoreo estándar en una planilla confeccionada para tal fin. Las variables hemodinámicas básicas de monitorización fueron la frecuencia cardíaca, la presión arterial media no invasiva (obtenida mediante esfigmomanómetro automático con un intervalo de 2 minutos entre mediciones consecutivas) y el trazado electrocardiográfico continuo en la derivación DIl. Luego en la etapa post intubación orotraqueal, los pacientes se mantuvieron recostados en posición supina y se ventilaron mecánicamente en modo volumen control usando el sistema Draeguer Fabius. Se estableció un volumen corriente de $8 \mathrm{ml} /$ $\mathrm{kg}$ de peso teórico, una frecuencia respiratoria de 14 ventilaciones por minuto, una presión positiva al final de la expiración de $3 \mathrm{~cm} \mathrm{H}_{2} \mathrm{O}$ y la presión pla-

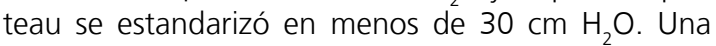
vez conectado el paciente a la ventilación mecánica, se registraron manualmente las variables de monitoreo hemodinámico mencionadas durante un período de 10 minutos. Durante el período de recolección de datos, fueron permitidos solamente procedimientos de estímulos bajos y moderados tales como la cateterización vesical y la preparación antiséptica del área quirúrgica.

\section{Ultrasonografía de la vena cava inferior ( $\mathrm{VCl}$ )}

Las mediciones de ultrasonidos se realizaron utilizando un ecógrafo SonoSite Micromaxx y el transductor transtorácico sectorial P17 de 5-1 MHz. Todas las medidas de $\mathrm{VCI}$ fueron realizadas por un operador con una experiencia de nivel 1 básico en ecocardiografía. La VCI se visualizó utilizando una visión paramediana de eje largo a través de un enfoque subxifoideo de acuerdo con la metodología descrita por la American Society of Echocardiography. Se obtuvo una imagen bidimensional de la $\mathrm{VCl}$ al entrar en la 
aurícula derecha. Se utilizó doppler de onda de pulso para diferenciar la VCI de la aorta. Las variaciones en el diámetro de la $\mathrm{VCl}$ con la respiración, se evaluaron utilizando la imagen en modo $\mathrm{M}$ y se calculó de 2 a $3 \mathrm{~cm}$ distal a la aurícula derecha. La imagen en modo $\mathrm{M}$ se generó a una velocidad de barrido medio. Para asegurar medidas consistentes de la $\mathrm{VCl}$, se realizaron tres exploraciones en cada paciente. Para cada individuo estudiado, se escogió la imagen de escaneo de mejor calidad. Los diámetros máximo y mínimo de VCl sobre un único ciclo respiratorio se midieron utilizando un software incorporado. El índice de colapsabilidad (IC) fue calculado según la siguiente fórmula y fue expresado como porcentaje.

$I C=\left(d V C I_{\max }-d V C I \min \right) / d V C I_{\max } * 100$

\section{Presión arterial no invasiva}

De acuerdo a numerosos ensayos clínicos previos, se consideró como episodio hipotensivo un valor de presión arterial media (PAM) menor de $60 \mathrm{mmHg}$ o una caída de $30 \%$ en la PAM con respecto a la medición basal. Al presentarse mencionados episodios, o si los mismos fueron prolongados (duración mayor o igual a 2 minutos), fueron tratados utilizando bolos de $1 \mathrm{ml}$ de etilefrina, a una dosis de $1 \mathrm{mg}$ por bolo administrado. La atropina debería haber sido administrada solo en caso de una bradicardia significativa (menos de 40 latidos por minutos), a una dosis de $0,01 \mathrm{mg} / \mathrm{kg}$.

\section{Manejo anestésico}

Se realizó premedicación en la sala de preanestesia con $0,03 \mathrm{mg} / \mathrm{kg}$ de midazolam intravenoso, $10 \mathrm{mi}$ nutos previos al acto quirúrgico. Una vez en quirófano se realizó preoxigenación con $\mathrm{O}_{2}$ al $100 \%$ durante 4 minutos. La inducción anestésica se realizó siguiendo un régimen protocolizado de 3-4 ug/kg de fentanilo, seguidos de $1,75 \mathrm{mg} / \mathrm{kg}$ de propofol. La intubación orotraqueal fue facilitada a través de la infusión de vecuronio a una dosis de $0,1 \mathrm{mg} / \mathrm{kg}$ y realizada $3 \mathrm{mi}-$ nutos posteriores a la inducción. El mantenimiento se realizó con sevoflurano al 1,5\% vehiculizado en aire enriquecido con oxígeno al 40\%. La velocidad de infusión de solución fisiológica fue de $5 \mathrm{ml} / \mathrm{kg} / \mathrm{h}$. Aquellos pacientes que experimentaron una instrumentación prolongada de la vía aérea fueron excluidos debido a la excesiva estimulación. Una vez recabados los datos hemodinámicos necesarios, se dio inicio a la cirugía y el manejo anestésico quedo a cargo y discreción del anestesiólogo tratante.

\section{Análisis estadístico}

Se presenta el promedio acompañado del desvío estándar (DE), además de los valores mínimo y máximo para describir las variables continuas y las frecuencias junto con los porcentajes para las variables categóricas. La comparación entre los grupos definidos según la presencia de hipotensión se realizó mediante test no paramétricos: el test $U$ de Mann-Whitney y el test de Fisher para las variables cuantitativas y para el género, respectivamente.

Se ajustó un modelo de regresión logística univariado para estudiar la capacidad predictiva del IC con respecto al desarrollo de hipotensión. Se ajustó, además, un modelo multivariado en el cual se consideraron como posibles factores de confusión la edad, el sexo, el IMC, la PAM y la FC basales y el porcentaje de cambio en la FC. Los resultados del ajuste se presentan mediante razones de Odds (RO) puntuales y con sus respectivos intervalos de confianza del 95\% $\left(\mathrm{IC}_{95 \%}\right)$. Se calcularon los valores de sensibilidad, especificidad, valor predictivo negativo (VPN) y valor predictivo positivo (VPP) para distintos puntos de corte en el IC de interés. El punto de corte óptimo se identificó como aquel que maximizó el índice de Youden (sensibilidad+ especificidad -1)[16].

El análisis estadístico se realizó mediante SAS/ STAT ${ }^{\circledR}$ software 9,3. Se consideró un nivel de significación de $5 \%$. A partir de los resultados hallados para los pacientes incluidos en el estudio, se determinó que el análisis se realizó con una potencia de $70 \%$.

\section{Resultados}

Se incluyeron en el estudio 80 pacientes, el $62,5 \%$ (50) de ellos eran mujeres y $37,5 \%$ (30) fueron hombres. Los pacientes presentaban una edad promedio igual a 42 años (DE: 12 años) y un IMC promedio igual a 26,6 (DE: 4,2) (Tabla 1). Excepto en la Tabla 1 , se presentan los datos de 78 individuos estudiados, debido a que en un caso se obtuvo una pobre visualización de la $\mathrm{VCl}$, y en el otro hubo una prolongada manipulación de la vía aérea superior y dificultad de intubación. Ningún paciente presentó una PAM menor de $70 \mathrm{mmHg}$. En ningún caso se observaron alteraciones en el trazado electrocardiográfico.

Luego de la inducción anestésica, 8 (10,3\%) pacientes desarrollaron hipotensión intraoperatoria de acuerdo a los criterios de nuestro estudio. Todos ellos recibieron el tratamiento propuesto con etilefrina endovenosa en bolo, a una dosis de $1 \mathrm{mg}$ por episodio hipotensivo desarrollado (Tabla 2). 


\begin{tabular}{lc}
\multicolumn{2}{c}{ Tabla 1. Características demográficas de los pacien- } \\
tes \\
\hline Características demográficas & $\mathbf{( n = 8 0 )}$ \\
Edad (años) ${ }^{\mathrm{a}}$ & $41,9(12,0)$ \\
& $(18-65)$ \\
Peso $(\mathrm{k})^{\mathrm{b}}$ & $75,0(13,7)$ \\
& $(45,0-107,0)$ \\
Talla $(\mathrm{m})^{\mathrm{b}}$ & $1,7(0,1)$ \\
& $(1,5-1,9)$ \\
IMC $\left(\mathrm{k} / \mathrm{m}^{2}\right)^{\mathrm{b}}$ & $26,6(4,2)$ \\
& $(18,7-35,0)$ \\
Sexo $(\text { femenino })^{\mathrm{b}}$ & $50(63 \%)$ \\
\hline
\end{tabular}

Los datos se presentan como: a promedio (desvío estándar) (mín. - máx.). ${ }^{\mathrm{b}} \mathrm{n}^{\circ}(\%)$.

El ajuste del modelo de regresión logística univariado para IC muestra una buena capacidad diagnóstica, siendo el área bajo la curva ROC igual a 0,76 (Figura 1). De acuerdo a dicho modelo, la chance de presentar hipotensión se incrementa en un $62 \%$ al aumentar el IC en 5 puntos. Por otro lado, si se interpreta el incremento del IC en 1 punto, el incremento en la chance de hipotensión es del 10\% ( $p=0,003)$.

El punto de corte óptimo del IC para predecir hipotensión se estima en $43 \%$ a partir de los datos analizados, con una sensibilidad de $62,5 \%$ y una especificidad de $92,9 \%$.

Se evaluó el efecto de las variables edad, IMC,

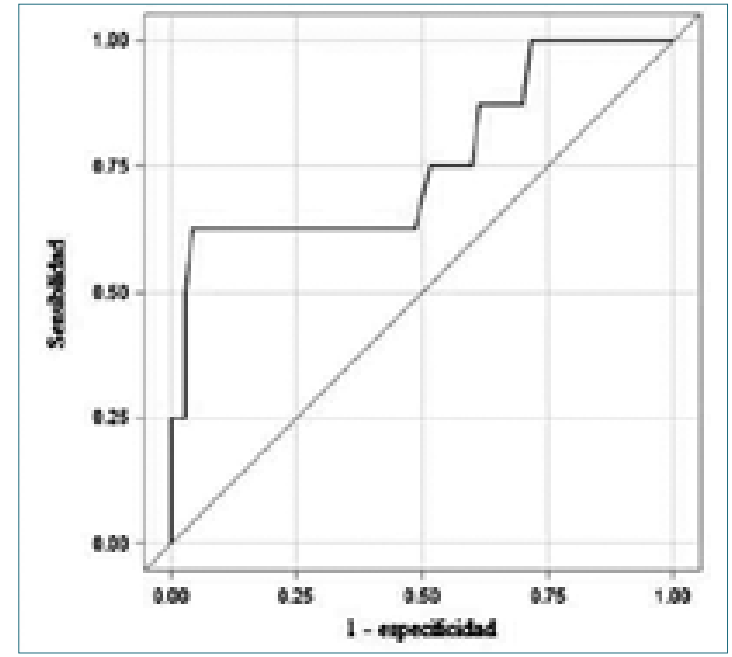

Figura 1. Curva ROC correspondiente al ajuste del modelo de regresión logística. Área bajo la curva ROC:0,76.

sexo, PAM basal, FC basal, y porcentaje de cambio en la FC en forma conjunta con el IC y no se encontraron resultados significativos (Tabla 3).

En cuanto al valor predictivo negativo, encontramos que con valores correspondientes a los puntos de corte entre $39 \%$ y $46 \%$, se obtenía un probabilidad de al menos 93,1\% de no presentar hipotensión (Tabla 4). En relación al valor predictivo positivo, éste fue igual a $41,7 \%$ para el punto de corte de $39 \%$ y de $50 \%$ para los dos puntos restantes.

\begin{tabular}{|c|c|c|c|}
\hline \multirow[t]{2}{*}{ Variable } & \multicolumn{2}{|c|}{ Presencia de hipotensión } & \multirow[t]{2}{*}{ Prob. asociada } \\
\hline & $\mathrm{Si}(\mathrm{n}=8)$ & No $(n=70)$ & \\
\hline Edad (años) ${ }^{\mathrm{a}}$ & $47,0(10,6)$ & $41,3(11,8)$ & 0,149 \\
\hline Peso $(k)^{a}$ & $83,8(13,5)$ & $73,8(13,6)$ & 0,093 \\
\hline Talla $(m)^{a}$ & $1,7(0,1)$ & $1,7(0,1)$ & 0,798 \\
\hline $\mathrm{IMC}\left(\mathrm{k} / \mathrm{m}^{2}\right)^{\mathrm{a}}$ & $29,6(3,8)$ & $26,2(4,1)$ & 0,025 \\
\hline Sexo ${ }^{b}$ & & & 0,246 \\
\hline Femenino & $7(14 \%)$ & $42(86 \%)$ & \\
\hline Masculino & $1(4 \%)$ & $28(96 \%)$ & \\
\hline IC $(\%)^{a}$ & $42,2(17,1)$ & $26,4(10,5)$ & 0,016 \\
\hline PAM basal $(\mathrm{mmHg})^{\mathrm{a}}$ & $105,8(20,2)$ & $98,0(14,0)$ & 0,378 \\
\hline FC basal (latidos por minuto) ${ }^{a}$ & $84,0(11,7)$ & $77,9(16,2)$ & 0,154 \\
\hline Pcte de cambio FC $(\%)^{a}$ & $-7,2(19,9)$ & $-5,1(10,5)$ & 0,639 \\
\hline
\end{tabular}

Los datos se presentan como: apromedio (desvío estándar) (mín. - máx.) - Test $U$ de Mann-Whitney. ${ }^{b} n^{\circ}(\%)$ - Test de Fisher. 
Tabla 3. Resultados del ajuste de un modelo de regresión logística multivariado para la evaluación de la presencia de hipotensión $(n=78)$

\begin{tabular}{lccc}
\hline \multicolumn{1}{c}{ Variable } & RO & \multicolumn{1}{c}{$\mathbf{I C}_{\text {RO95\% }}$} & 1,19 \\
IC & 1,10 & 1,02 & 1,14 \\
Edad & 1,04 & 0,94 & 1,67 \\
IMC & 1,26 & 0,95 & 5,44 \\
Sexo & 0,43 & 0,03 & 1,05 \\
PAM basal & 0,98 & 0,91 & 1,12 \\
FC basal & 1,04 & 0,96 & 1,09 \\
Pcte de cambio FC & 1,01 & 0,93 & \\
\hline
\end{tabular}

a Referencia: femenino.

Tabla 4. Sensibilidad, especificidad, VPN y VPP para distintos puntos de corte en el valor del IC a partir del modelo univariado

\begin{tabular}{cccc}
\hline Punto de corte en el IC & Sensibilidad & Especificidad & VPN \\
$39 \%$ & $62,5 \%$ & $90,0 \%$ & $95,5 \%$ \\
$43 \%$ & $62,5 \%$ & $92,9 \%$ & $95,6 \%$ \\
$46 \%$ & $37,5 \%$ & $95,7 \%$ & $93,1 \%$ \\
\hline
\end{tabular}

\section{Discusión}

En la actualidad, el uso de la ultrasonografía de la VCl como herramienta para evaluar la volemia preoperatoria y como guía en la terapéutica de fluidos no se encuentra bien definido. En este trabajo, la medición de la VCI por ultrasonido y el cálculo del IC, previa a la inducción de anestesia general, mostró una buena capacidad para predecir la presencia de hipotensión intraquirúgica posterior. A su vez, las pautas de la American Society of Echocardiography respaldan el uso del IC en la evaluación del estado de la volemia[17], Wallace et al[18], encontraron que el IC se vio afectado por la ubicación del muestreo. En este estudio, limitamos el muestreo a 2 a $3 \mathrm{~cm}$ distal a la entrada de la VCI en la aurícula derecha. Clínicamente, las medidas de IC preoperatorias fueron fáciles y rápidas de obtener (tiempo de exploración de menos de $10 \mathrm{~min}$ ). Nuestra tasa de fracaso de la exploración de la VCI fue de 1,25\% y por tanto, una tasa de éxito de $98,75 \%$ para la evaluación de la misma. Este hecho aparentemente se debería a la inclusión de pacientes con un IMC menor a 35.

Nuestros resultados indicaron que los valores de corte para el IC, correspondientes al 39\% y $43 \%$, se asociaron con un alto valor predictivo negativo y predijeron estabilidad hemodinámica luego de la inducción anestésica. Un IC menor al 43\% fue predictivo, con alta especificidad (92,9\%) y moderada sensibilidad (62,5\%), del menor riesgo de desarrollar un episodio hipotensivo posinducción. En la misma línea de estudio, Zhang et al.[14], describieron que un IC de más de $43 \%$ fue altamente predictivo, con alta especificidad $(91,7 \%)$ y sensibilidad moderada $(78,6 \%)$ de desarrollar hipotensión intraoperatoria posinducción.

El IC fue un predictor independiente de hipotensión posterior a la inducción luego de ajustar por edad, ASA, IMC y PAM basal. Si bien el estudio de Reich et al.[19], encontraron que la vejez es un predictor significativo de hipotensión después de la inducción, no fue un predictor significativo en el estudio actual. Esto puede deberse al pequeño tamaño de la muestra y al grupo etario incluido.

Aunque la hipotensión intraoperatoria es un efecto secundario frecuente de la anestesia, su definición varía entre los numerosos estudios clínicos. En este sentido, Bijker et al.[20], encontraron 140 definiciones en la literatura, lo que resulta en diferentes incidencias reportadas de hipotensión. En este trabajo se estableció una disminución de la PAM superior a 30\% desde el inicio o una PAM menor de $60 \mathrm{mmHg}$ como definición de hipotensión. Nuestro período de estudio fue desde la inducción hasta 10 minutos después de 
Índice de colapsabilidad de la vena cava inferior como predictor de hipotensión intraoperatoria - M.C. Rossi et al.

la intubación traqueal. La incidencia de hipotensión fue de $10,3 \%$.

Evaluar el estado del volumen intravascular debería ser un enfoque diagnóstico primario. La inclusión del ultrasonido perioperatorio de la $\mathrm{VCl}$ ayudaría a identificar aquellos pacientes que se beneficiarían de una optimización de su volemia para así disminuir el riesgo de desarrollar hipotensión y la consecuente injuria por isquemia-reperfusión.

Estos resultados demuestran que las variaciones respiratorias del diámetro de la $\mathrm{VCl}$, predicen la incidencia de hipotensión luego de la anestesia general con un alto grado de sensibilidad y especificidad. Las futuras investigaciones clínicas deberían centrarse en la efectividad de la terapéutica con líquidos intravenosos, drogas inotrópicas y/o vasoconstrictoras, sobre todo cuando las lecturas ultrasonográficas del IC alertan sobre la posibilidad de hipotensión posinducción.

\section{Conclusión}

Los resultados obtenidos en este trabajo indican que la ecografía de la VCI y la medida del IC preoperatoriamente, proporcionan un predictor confiable de hipotensión después de la inducción de anestesia general. Un menor IC se asoció con una baja chance de hipotensión intraoperatoria. Por lo tanto, el IC puede proporcionar información útil para guiar nuestra terapéutica y disminuir así las complicaciones derivadas de la hipovolemia e injuria por isquemiareperfusión.

\section{Referencias}

1. Barbier $C$, Loubières $Y$, Schmit C, Hayon J, Ricôme JL, Jardin $\mathrm{F}$, et al. Respiratory changes in inferior vena cava diameter are helpful in predicting fluid responsiveness in ventilated septic patients. Intensive Care Med. 2004 Sep;30(9):1740-6. https:// doi.org/10.1007/s00134-0042259-8 PMID:15034650

2. Feissel M, Michard F, Faller JP, Teboul JL. The respiratory variation in inferior vena cava diameter as a guide to fluid therapy. Intensive Care Med. 2004 Sep;30(9):1834-7. https://doi. org/10.1007/s00134-004-22335 PMID:15045170

3. Duwat A, Zogheib E, Guinot $P$, Levy F, Trojette F, Diouf M, et al. The gray zone of the qualitative assessment of respiratory changes in inferior vena cava diameter in ICU patients. Crit Care. 2014 Jan;18(1):R14. https://doi.org/10.1186/cc13693 PMID:24423180

4. Via G, Tavazzi G, Price S. Ten situations where inferior vena cava ultrasound may fail to accurately predict fluid responsiveness: a physiologically based point of view. Intensive Care Med. 2016 Jul;42(7):1164-7. https://doi. org/10.1007/s00134-016-43579 PMID:27107754

5. Nakamura K, Tomida M, Ando T, Sen K, Inokuchi R, Kobayashi $\mathrm{E}$, et al. Ando T y cols. Cardiac variation of inferior vena cava: new concept in the evaluation of intravascular blood volume. J Med Ultrason. 2013;40(3):205-9. https://doi.org/10.1007/s10396013-0435-6.

6. Dipti A, Soucy Z, Surana A, Chandra S. Role of inferior vena cava diameter in assessment of volume status: a meta-analysis. Am J Emerg Med. 2012 Oct;30(8):14141419.e1. https://doi. org/10.1016/j.ajem.2011.10.017 PMID:22221934

7. Zhang Z, Xu X, Ye S, Xu L. Ultrasonographic measurement of the respiratory variation in the inferior vena cava diameter is predictive of fluid responsiveness in critically ill patients: systematic review and meta-analysis. Ultrasound Med Biol. 2014 May;40(5):84553. https://doi.org/10.1016/j. ultrasmedbio.2013.12.010 PMID:24495437

8. Kent A, Bahner DP, Boulger CT, Eiferman DS, Adkins EJ, Evans DC, et al. Sonographic evaluation of intravascular volume status in the surgical intensive care unit: a prospective comparison of subclavian vein and inferior vena cava collapsibility index. J Surg Res. 2013 Sep;184(1):5616. https://doi.org/10.1016/j. jss.2013.05.040 PMID:23764308

9. Sabatiera C, Mongeb I, Maynarc J, Ochagavia A. Valoración de la precarga y la respuesta cardiovascular al aporte de volumen. Med Intensiva. 2012;36(1):4555. https://doi.org/10.1016/j. medin.2011.04.005.

10. Corl K, Napoli AM, Gardiner F. Bedside sonographic measurement of the inferior vena cava caval index is a poor predictor of fluid responsiveness in emergency department patients. Emerg Med Australas. 2012 Oct;24(5):534-9. https://doi.org/10.1111/ j.1742-6723.2012.01596.x PMID:23039295

11. M. Lyon N. Verma. Ultrasound guided volume assessment using 
inferior vena cava diameter. The Open Emergency Medicine Journal 2010 3(1):22-24 • https://doi./10 2174/1876542401003010022

12. Walsh M, Devereaux PJ, Garg AX, Kurz A, Turan A, Rodseth $\mathrm{RN}$, et al. Relationship between intraoperative mean arterial pressure and clinical outcomes after noncardiac surgery: toward an empirical definition of hypotension. Anesthesiology. 2013 Sep;119(3):50715. https://doi.org/10.1097/ ALN.0b013e3182a10e26 PMID:23835589

13. David L. Reich, MD, Sabera Hossain, MA, Marina Krol, PhD, Bernard Baez, MD, Puja Patel. Predictors of Hypotension After Induction of General Anesthesia. Anesth Analg. 2005;101:622-8.

14. Zhang J, Critchley LA. Inferior Vena Cava Ultrasonography before General Anesthesia Can Predict Hypotension after Induction. Anesthesiology. 2016 Mar;124(3):580-9. https://doi.org/10.1097/ ALN.0000000000001002
PMID:26771910

15. Do Pico JL, Parra G, Puppo C. Ultrasonografia en el paciente crítico. 1st ed. Buenos Aires: Journal; 2014. pp. 198-03.

16. Youden WJ. Index for rating diagnostic tests. Cancer. 1950 Jan;3(1):32-5. https:// doi.org/10.1002/10970142(1950)3:13.0.CO;2-3 PMID:15405679

17. Rudski LG, Lai WW, Afilalo J, Hua L, Handschumacher MD, Chandrasekaran $\mathrm{K}$, et al. Guidelines for the echocardiographic assessment of the right heart in adults: a report from the American Society of Echocardiography endorsed by the European Association of Echocardiography, a registered branch of the European Society of Cardiology, and the Canadian Society of Echocardiography. J Am Soc Echocardiogr. 2010 Jul;23(7):685-713. https://doi. org/10.1016/j.echo.2010.05.010 PMID:20620859

18. Wallace DJ, Allison M, Stone $\mathrm{MB}$. Inferior vena cava percen- tage collapse during respiration is affected by the sampling location: an ultrasound study in healthy volunteers. Acad Emerg Med. 2010 Jan;17(1):969. https://doi.org/10.1111/ j.1553-2712.2009.00627.x PMID:20003120

19. Reich DL, Hossain S, Krol M, Baez B, Patel P, Bernstein $A$, et al. Predictors of hypotension after induction of general anesthesia. Anesth Analg. 2005 Sep;101(3):622-

8. https://doi.org/10.1213/01. ANE.0000175214.38450.91 PMID:16115962

20. Bijker JB, van Klei WA, Kappen $\mathrm{TH}$, van Wolfswinkel L, Moons KG, Kalkman CJ. Incidence of intraoperative hypotension as a function of the chosen definition: literature definitions applied to a retrospective cohort using automated data collection. Anesthesiology. 2007 Aug;107(2):213-20. https://doi.org/10.1097/01. anes.0000270724.40897.8e PMID:17667564 